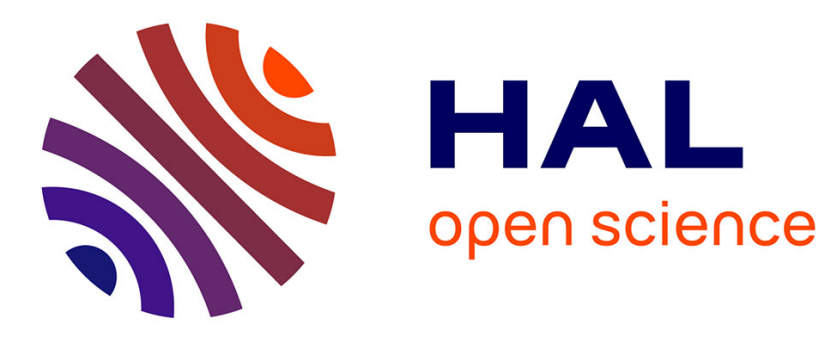

\title{
Scheduling Real-time Mixed-criticality Jobs
}

Sanjoy K. Baruah, Vincenzo Bonifaci, Gianlorenzo d'Angelo, Haohan Li, Alberto Marchetti-Spaccamela, Nicole Megow, Leen Stougie

\section{To cite this version:}

Sanjoy K. Baruah, Vincenzo Bonifaci, Gianlorenzo d'Angelo, Haohan Li, Alberto MarchettiSpaccamela, et al.. Scheduling Real-time Mixed-criticality Jobs. IEEE Transactions on Computers, 2012, 61 (8), pp.1140-1152. 10.1109/TC.2011.142 . hal-00643942

\section{HAL Id: hal-00643942 \\ https://hal.inria.fr/hal-00643942}

Submitted on 23 Nov 2011

HAL is a multi-disciplinary open access archive for the deposit and dissemination of scientific research documents, whether they are published or not. The documents may come from teaching and research institutions in France or abroad, or from public or private research centers.
L'archive ouverte pluridisciplinaire HAL, est destinée au dépôt et à la diffusion de documents scientifiques de niveau recherche, publiés ou non, émanant des établissements d'enseignement et de recherche français ou étrangers, des laboratoires publics ou privés. 


\title{
Scheduling real-time mixed-criticality jobs
}

\author{
Sanjoy Baruah, Vincenzo Bonifaci, Gianlorenzo D’Angelo, Haohan Li, \\ Alberto Marchetti-Spaccamela, Nicole Megow, and Leen Stougie
}

\begin{abstract}
Many safety-critical embedded systems are subject to certification requirements; some systems may be required to meet multiple sets of certification requirements, from different certification authorities. Certification requirements in such "mixed-criticality" systems give rise to interesting scheduling problems, that cannot be satisfactorily addressed using techniques from conventional scheduling theory. In this paper, we study a formal model for representing such mixed-criticality workloads. We demonstrate first the intractability of determining whether a system specified in this model can be scheduled to meet all its certification requirements, even for systems subject to merely two sets of certification requirements. Then we quantify, via the metric of processor speedup factor, the effectiveness of two techniques, reservation-based scheduling and priority-based scheduling, that are widely used in scheduling such mixed-criticality systems, showing that the latter of the two is superior to the former. We also show that the speedup factors we obtain are tight for these two techniques.
\end{abstract}

\section{INTRODUCTION}

Due to considerations of cost, energy efficiency, thermal dissipation, etc., there is an increasing trend in embedded systems towards implementing multiple functionalities upon a single shared

Some preliminary results and the first parts of Theorems 6 and 7 in this paper have been published in conference proceedings [5]-[7]

Baruah and Li are with the Department of Computer Science, The University of North Carolina at Chapel Hill, USA (\{baruah,lihaohan\}@cs.unc.edu).

Bonifaci and Megow are with Max-Planck-Institut für Informatik, Saarbrücken, Germany (\{bonifaci,nmegow\}@mpiinf.mpg.de).

D’Angelo is with Università degli Studi dell'Aquila, Monteluco di Roio, Italy (gianlorenzo.dangelo@univaq.it).

Marchetti-Spaccamela is with Sapienza University of Rome, Rome, Italy (alberto@ dis.uniroma1.it).

Stougie is with Division of Operations Research, Dept. of Economics and Business Administration, Vrije Universiteit, and CWI, Amsterdam, The Netherlands (lstougie@ feweb.vu.nl). 
computing platform. The concept of mixed criticalities is fast coming to be regarded as an important concept in such systems. (Indeed, mixed criticalities has been identified as one of the core foundational concepts in the emerging discipline of Cyber Physical Systems.) In such systems, mixed criticalities can mean two different things. The first meaning is the obvious one: upon platforms that offer support for multiple functionalities, it is highly likely that some of these functionalities will be more important (more "critical") to the overall welfare of the platform than others. For instance, it is more important to the correct behavior of an automotive control system that the anti-lock brake system (ABS) works correctly than that the on-board radio does so. This aspect of mixed criticalities is widely studied by embedded systems designers, who typically address such differences in criticalities by means of priority-based scheduling approaches: more critical functionalities are accorded greater priority so that they will be less likely to suffer performance degradation in the event of system overload .

However, there is another aspect [4] to mixed criticalities that arises in application domains (such as civilian and defense avionics) that are subject to mandatory certification requirements by statutory organizations. Coming up with procedures that will allow for the cost-effective certification of such mixed-criticality systems has been recognized as a unique, particularly challenging, collection of problems [4]. Recognizing these challenges, several US government R\&D organizations including the Air Force Research Laboratory, the National Science Foundation, the National Security Agency, the National Aeronautics and Space Administration, etc., have led initiatives such as the Mixed Criticality Architecture Requirements (MCAR) program aimed at streamlining the certification process for safety-critical embedded systems; these initiatives have brought together participants from industry, academia, and standards bodies to seek out more advanced, efficient, and cost-effective certification processes. Within this setting, new interesting scheduling problems arise that are the focus of this paper.

We introduce these problems by considering first an example from the domain of unmanned aerial vehicles (UAV's), used for defense reconnaissance and surveillance. The functionalities on board such UAV's may be classified into two levels of criticality:

- Level 1: the mission-critical functionalities, concerning reconnaissance and surveillance objectives, like capturing images from the ground, transmitting these images to the base station, etc.

- Level 2: the flight-critical functionalities: to be performed by the aircraft to ensure its safe 
operation.

For permission to operate such UAV's over civilian airspace (e.g., for border surveillance), it is mandatory that its flight-critical functionalities be certified correct by civilian Certification Authorities (CA's) such as the US Federal Aviation Authority (FAA), which tend to be very conservative concerning the safety requirements. However, these CA's are not concerned with the mission-critical functionalities: these must be validated separately by the system designers (and presumably the customers - those who will purchase the aircraft). The latter are also interested in ensuring the correctness of the flight-critical functionalities, but the notion of correctness adopted in validating these functionalities is typically less rigorous than the one used by the civilian CA's.

This difference in correctness criteria may be expressed by different Worst-Case Execution Times (WCET) estimates for the execution of a piece of real-time code. In fact, the CA and the system designers (and other parties responsible for validating the mission-critical functionalities) will each have their own tools, rules, etc., for estimating WCET; the value so obtained by the $\mathrm{CA}$ is likely to be larger (more pessimistic) than the one obtained by the system designer. We illustrate via a (contrived) example.

Example 1: Consider a system comprised of two jobs: $J_{1}$ is flight-critical while $J_{2}$ has lower mission-critical criticality. Both jobs arrive at time-instant 0 , and have their deadlines at timeinstant 10. For $i \in\{1,2\}$, let $P_{i}(1)$ denote the WCET estimate of job $J_{i}$ as made by the system designer, and $P_{i}(2)$ the WCET estimate of job $J_{i}$ as made by the CA.

Suppose that $P_{1}(1)=3, P_{1}(2)=5$ and $P_{2}(1)=P_{2}(2)=6$. Consider the schedule that first executes $J_{1}$ and then $J_{2}$.

- The CA responsible for safety-critical certification would determine that $J_{1}$ completes latest by time-instant 5 and meets its deadline. (Note that if the execution time of $J_{1}$ is 5 then in the worst case it is not possible to complete $J_{2}$ by its deadline; however, this CA is not interested in $J_{2}$; hence the system passes certification.)

- The system designers (and other parties responsible for validating the correctness of the mission-critical functionalities) determine that $J_{1}$ completes latest by time-instant 3 , and $J_{2}$ by time-instant 9 . Since both jobs complete by their deadlines, the system is determined to be correct by its designers.

We thus see that the system is deemed as being correct by both the CA and the designers, 
despite the fact that the sum of the WCET's of the jobs at their own criticality levels (6 and 5) exceeds the length of the scheduling window over which they are to execute.

Current practice in safety-critical embedded systems design for certifiability is centered around the technique of "space-time partitioning," as codified in, e.g., the ARINC-653 standard [1], [16]. Loosely speaking, space partitioning means that each application is granted exclusive access to some of the physical resources on board the platform, and time partitioning means that the time-line is divided into slots with each slot being granted exclusively to some (pre-specified) application. Interactions among the partitioned applications may only occur through a severely limited collection of carefully-designed library routines. This is one of several reservation-based approaches, in which a certain amount of the capacity of the shared platform is reserved for each application, that have been considered for designing certifiable mixed-criticality systems. It is known that reservation-based approaches tend to be pessimistic (in the sense of underutilizing platform resource); for instance, a reservation-based approach to the example above would require that 5 units of execution be reserved for job $J_{1}$, and 6 units for job $J_{2}$, over the interval $[0,10)$.

The central thesis explored in our research, as illustrated in Example 1, is that efficient resource utilization in systems that are subject to multiple different correctness criteria requires the development of new approaches for resource-allocation and scheduling. This paper describes our efforts to date towards developing such approaches.

1) We have proposed [5], [6] a formal model for representing mixed-criticality real-time systems - this mixed-criticality (MC) model extends the conventional model of a real-time job by allowing for the specification of different WCET's for a job at different criticality levels. This model is described in Section II.

2) We have studied the computational complexity of mixed-criticality scheduling problems. In previous papers [5], [6], the problem of deciding schedulability of a given MC system was conjectured to be NP-hard. We provide a proof of this result here, in Section III. However, the exact complexity of the problem remains open, since it is not clear whether the problem is actually in NP. We prove that it is, if the number of criticality levels is a constant. Otherwise, we can only show that it is in PSPACE. In the same section we present an algorithm that decides MC-schedulability efficiently for the special case in which all jobs 
have the same deadline.

3) In Section IV we quantitatively evaluate, via the metric of processor speedup factor (cf. resource augmentation in performance analysis of approximation algorithms, as initiated in [10]), two techniques that are currently widely used for resource-allocation and scheduling in mixed criticality systems subject to certification. Our results here extend the results in [6], which considered the techniques for dual-criticality systems, in which there are only two different criticality levels (as in Example 1 above). Our results improve the results in [5], where also the techniques for $L$ criticality levels are studied. Moreover, we prove here that our results are tight.

4) In Section V we show that the bounds of Section IV do not hold for restricted classes of mixed-criticality systems. We focus on systems in which the range of WCET parameter estimate values is restricted. More specifically, we consider mixed-criticality systems in which there are at most two distinct criticality levels (as in Example 1 above), and we assume that the sum of the level 2 WCET estimates is bounded by a constant $\beta$ times the sum of the level 1 WCET estimates of these jobs. We show that, depending on $\beta$, performance guarantees can be made that are superior to the tight bounds of the general case.

\section{PRELIMINARIES}

Although the examples that we considered in Section I were characterized by just two criticality levels, systems may in general have more criticality levels defined. (For instance, the RTCA DO178-B standard, widely used in the aviation industry, specifies 5 different criticality levels, with the system designer expected to assign one of these criticality levels to each job. The ISO 26262 standard, used in the automotive domain, specifies 4 criticality levels, known in the standard as "safety integrity levels" or SIL's.)

Accordingly, the formal model that we propose allows for the specification of arbitrarily many criticality levels. Let $L \in \mathbb{N}^{+}$denote the number of distinct criticality levels in the MC system being modeled. A job in this MC system is characterized by a 4-tuple of parameters: $J_{j}=$ $\left(r_{j}, d_{j}, \chi_{j}, P_{j}\right)$, where

- $r_{j} \in \mathbb{Q}_{+}$is the release time;

- $d_{j} \in \mathbb{Q}_{+}$is the deadline, $d_{j} \geq r_{j}$; 
- $\chi_{j} \in \mathbb{N}_{+}$is the criticality of the job;

- $P_{j} \in \mathbb{Q}_{+}^{L}$ is a vector, the $\ell$-th coordinate of which specifies the worst-case execution time (WCET) estimate of job $J_{j}$ at criticality level $\ell$. In a job-specification we will usually represent it by $\left(P_{j}(1), \ldots, P_{j}(L)\right)$.

We will, for the most part, assume that $P_{j}(\ell)$ is monotonically non-decreasing with increasing $\ell$. This is a reasonable assumption: these $P_{j}(\ell)$ values represent upper bounds, at different degrees of confidence, on the WCET of the job. Larger values of $\ell$ correspond to greater degrees of confidence, and are therefore likely to be larger. At any moment, we call a job available if its release time has passed and the job has not yet completed execution.

An instance $I$ of the MC-schedulability problem consists of a set of $n$ jobs. In this paper we assume that there is only one machine (processor) to execute the jobs. We assume that this processor is preemptive: executing jobs may have their execution interrupted at any instant in time and resumed later, with no additional cost or penalty.

To define MC-schedulability we define the notion of a scenario. Each job $J_{j}$ requires an amount of execution time $p_{j}$ within its time window $\left[r_{j}, d_{j}\right]$. The value of $p_{j}$ is not known from the specification of $J_{j}$, but is only discovered by actually executing the job until it signals that it has completed execution. This characterizes the uncertainty of the problem. We call a collection of realized values $\left(p_{1}, p_{2}, \ldots, p_{n}\right)$ a scenario of instance $I$.

We define the criticality level, or simply criticality, of a scenario $\left(p_{1}, p_{2}, \ldots, p_{n}\right)$ of $I$ as the smallest integer $\ell$ such that $p_{j} \leq P_{j}(\ell)$ for all $j=1, \ldots, n$. (If there is no such $\ell$, we define that scenario to be erroneous.)

Definition 1: A schedule for a scenario $\left(p_{1}, \ldots, p_{n}\right)$ of criticality $\ell$ is feasible if every job $J_{j}$ with $\chi_{j} \geq \ell$ receives execution time $p_{j}$ during its time window $\left[r_{j}, d_{j}\right]$.

A clairvoyant scheduling policy knows the scenario of $I$, i.e., $\left(p_{1}, \ldots, p_{n}\right)$, prior to determining a schedule for $I$.

Definition 2: An instance $I$ is clairvoyantly-schedulable if for each non-erroneous scenario of $I$ there exists a feasible schedule.

By contrast, an on-line scheduling policy discovers the value of $p_{j}$ only by executing $J_{j}$ until it signals completion. In particular, the criticality level of the scenario becomes known only by executing jobs. At each time instant, scheduling decisions can be based only on the partial information revealed thus far. 
Definition 3: An on-line scheduling policy is correct for instance $I$ if for any non-erroneous scenario of instance $I$ the policy generates a feasible schedule.

Definition 4: An instance $I$ is $M C$-schedulable if it admits a correct on-line scheduling policy.

The MC-SChedulability problem is to determine whether a given instance $I$ is $\mathrm{MC}$ schedulable or not. The following is obvious.

Proposition 1: If an instance $I$ is MC-schedulable on a given processor, then $I$ is clairvoyantlyschedulable on the same processor.

Example 2: Consider an instance $I$ of a dual-criticality system: a system with $L=2$. $I$ is comprised of 2 jobs: job $J_{1}$ has criticality level 1 (which is the lower criticality level), and the other job has the higher criticality level 2.

$$
\begin{aligned}
& J_{1}=(0,2,2,(1,1)) \\
& J_{2}=(0,3,2,(1,3))
\end{aligned}
$$

For this example instance, any scenario in which $p_{1}$ and $p_{2}$, are no larger than 1 , has criticality 1; while any scenario not of criticality 1 in which $p_{1}$ and $p_{2}$ are no larger than 1 , and 3 , respectively, has criticality 2 . All remaining scenarios are, by definition, erroneous. It is easy to verify that this instance is clairvoyantly-schedulable.

Policy $S_{0}$, described below, is an example of an on-line scheduling policy for instance $I$ :

$S_{0}$ : Execute $J_{2}$ over [0,1]. If $J_{2}$ has no remaining execution (i.e., $p_{2}$ is revealed to be no greater than 1$)$, then continue with scheduling $J_{1}$ over $(1,2]$; else continue by complete scheduling $J_{2}$.

It is easy to see that policy $S_{0}$ is correct for instance $I$. However, $S_{0}$ is not correct if we modify the deadline of $J_{1}$ obtaining the following instance $I^{\prime}$ :

$$
\begin{aligned}
& J_{1}=(0,1,2,(1,1)) \\
& J_{2}=(0,3,2,(1,3))
\end{aligned}
$$

It is easy to see that $I^{\prime}$ is clairvoyantly schedulable but not MC-schedulable.

The above example shows that there are instances that are clairvoyantly schedulable but not MC-schedulable. Indeed, this is true even if the machine upon which the on-line algorithm 
executes is faster than the one upon which the clairvoyant scheduler executes ${ }^{1}$. This is shown in the following proposition.

Proposition 2: There are dual-criticality instances that are clairvoyantly schedulable on a given processor, but that are not MC-schedulable on a processor that is less than $(1+\sqrt{5}) / 2$ times as fast.

Proof: Let $\sigma=(1+\sqrt{5}) / 2$ and consider the following instance

- $J_{1}=(0,1,1,(1,1))$;

- $J_{2}=(0, \sigma, 2,(\sigma-1, \sigma))$.

This system is clairvoyantly schedulable. To analyze its MC-schedulability, consider the possible policies on a higher speed- $s$ processor. The first one starts with $J_{2}$ and runs it till $P_{2}(1)=(\sigma-$ $1) / s$, and if it signals completion, schedule $J_{1}$ which then finishes latest by $(\sigma-1) / s+1 / s=\sigma / s$. This is feasible only if $\sigma / s \leq 1$, that is, $s \geq \sigma$. The other policy is simply to first schedule $J_{1}$ and then $J_{2}$, which may require a total execution time $1 / s+\sigma / s$, which is feasible only if $(1+\sigma) / s \leq$ $\sigma$, that is, $s \geq(\sigma+1) / \sigma$. Hence, if the processor has speed $s<\min \{\sigma,(\sigma+1) / \sigma\}$, neither of the possible scheduling policies is correct. Taking $\sigma=(\sigma+1) / \sigma$, that is, $\sigma=(1+\sqrt{5}) / 2$, implies $s \geq \sigma$.

\section{COMPleXity OF MC-SChEDUlability}

In this section we investigate the complexity of MC-SCHEDULABILITY. We show that it is NP-hard in the strong sense. However, a little thought should make it clear that it is not trivial to decide if the problem belongs to NP or not. We prove that it actually belongs to NP if the number of criticality levels is bounded by a fixed constant. For the general case, in which the number of criticality levels is part of the input, we show that it belongs to the class PSPACE, leaving membership to NP as an open question. We complete the section by presenting a well-solved special case of MC-SCHEDULABILITY, in which all jobs have equal deadline.

A preliminary observation is that determining clairvoyant-schedulability has the same complexity as the ordinary scheduling problem with only 1 criticality level: verify for each criticality

\footnotetext{
${ }^{1}$ This notion, of comparing the performance of an on-line algorithm executing upon a faster processor than the processor available to the clairvoyant algorithm, is formalized in the concept of resource augmentation; this concept is explored further in Section IV.
} 
level $\ell=1, \ldots, L$ if the jobs of that criticality level or higher can be scheduled to complete before their deadlines if each such job $j$ has execution time $P_{j}(\ell)$. In particular this means that clairvoyant-schedulability of any instance on a fully preemptive processor platform can be verified in polynomial time. This also holds if $P_{j}(\ell)$ is not monotonic in $\ell$.

We show that it is strongly NP-hard to determine whether a given clairvoyantly-schedulable system is also MC-schedulable upon a fully preemptive single-processor platform.

Theorem 1: MC-SCHEDULABILITY is NP-hard in the strong sense, even when all release times are identical and there are only two criticality levels.

Proof: The proof is by reduction from the strongly NP-complete problem 3-PARTITION [9]. In an instance $I_{3 P}$ of 3-PARTITION, we are given a set $S$ of $3 m$ positive integers $s_{0}, s_{1}, \ldots, s_{3 m-1}$ and a positive integer $B$ such that $B / 4<s_{i}<B / 2$ for each $i$ and $\sum_{i=0}^{3 m-1} s_{i}=m B$. The problem is to decide whether $S$ can be partitioned into $m$ disjoint sets $S_{0}, S_{1}, \ldots, S_{m-1}$ such that, for $0 \leq k<m, \sum_{s_{i} \in S_{k}} s_{i}=B$.

From a given instance $I_{3 P}$ we construct an MC-SCHEDULABILITY instance $I_{M C}$ consisting of $4 m$ jobs with release time 0 , which in the 4-tuple notation are:

- 3P-jobs: For each $i, 0 \leq i<3 m$, job $J_{i}=\left(0,2 m B, 2,\left(s_{i}, 2 s_{i}\right)\right)$;

- Blocking jobs: For each $k, 0 \leq k<m$, job $J_{3 m+k}=(0,2(k+1) B, 1,(B, B))$.

Clairvoyant-schedulability can be verified easily. In each scenario of criticality level 2 , the blocking jobs do not need to be scheduled, and the total execution time of the 3P-jobs is at most $\sum_{i=0}^{3 m-1} P_{i}(2)=\sum_{i=0}^{3 m-1} 2 s_{i}=2 m B$. For each scenario of criticality level 1, each blocking job is scheduled for at most $B$ time-units immediately preceding its deadline. The total available execution time before the common deadline $2 m B$ of the $3 \mathrm{P}$-jobs is at least $m B \geq \sum_{i=0}^{3 m-1} P_{i}(1)$.

We show that instance $I_{3 P}$ is a YES-instance of 3-PARTITION if and only if the corresponding instance $I_{M C}$ is MC-schedulable.

Suppose there is a feasible partition $S_{0}, S_{1}, \ldots, S_{m-1}$ for instance $I_{3 P}$. Based on this partition, a feasible online scheduling policy for jobs in $I_{M C}$ is as follows. For each $k=0,1, \ldots, m-$ 1 , reserve, for the 3P-jobs $J_{i}$ corresponding to each $s_{i} \in S_{k}, P_{i}(1)=s_{i}$ units in the timeinterval $[2 k B,(2 k+1) B)$. If all jobs associated with a set $S_{k}$ signal that they have completed execution in this time interval, then schedule the blocking job $J_{3 m+k}$ over the interval $[(2 k+$ 1) $B,(2 k+2) B)$. Else, discard job $J_{3 m+k}$ and complete the execution of the jobs that had not completed. Note that this is possible since $\sum_{i \mid s_{i} \in S_{k}} P_{i}(2)=2 B$ which is equal to the length of 
the interval $[2 k B,(2 k+2) B)$, which is now completely available for execution of 3P-jobs.

Suppose that there is no 3-partition for instance $I_{3 P}$. Consider any online policy. Since preemption is allowed, we can assume w.l.o.g. that the policy schedules each blocking job $J_{3 m+k}$ as late possible, that is, in intervals $[(2 k+1) B, 2(k+1) B), k=0,1, \ldots, m-1$, as long as the system's scenario is in criticality level 1. Take a scenario in which each job needs either $P_{i}(1)$ or $P_{i}(2)$. Let $k^{\prime}$ be the smallest value of $k$ for which there exists a 3P-job $J_{j}$ which receives execution time $p_{j}^{\prime}$, with $0<p_{j}^{\prime}<P_{j}(1)$, during the interval $\left[0,\left(2 k^{\prime}+1\right) B\right)$, and such that the scenario is still of criticality level 1 at time $\left(2 k^{\prime}+1\right) B$. Because there is no 3-partition, a scenario must exist such that $k^{\prime}$ and $J_{j}$ exist. Since the system's scenario is still at criticality level 1 , the online policy must schedule a blocking job at $\left[\left(2 k^{\prime}+1\right) B, 2\left(k^{\prime}+1\right) B\right)$.

Now, let any 3P-job $J_{i}$ be scheduled after time $2\left(k^{\prime}+1\right) B$ report an execution time of $P_{i}(2)$. Clearly, any sensible online policy discards all blocking jobs $J_{3 m+k}$ with $k>k^{\prime}$, and uses all the remaining available time up to the common deadline of the $3 \mathrm{P}$-jobs, that is, $2 m B-2\left(k^{\prime}+\right.$ 1) $B$, for processing $3 \mathrm{P}$-jobs. However, the total remaining processing requirement of these jobs, is $2\left(m B-\left(k^{\prime}+1\right) B\right)+p_{j}^{\prime}>2 m B-2\left(k^{\prime}+1\right) B$.

The question remains as to whether MC-SCHEDULABILITY belongs to the complexity class NP or not. In case the number of criticality levels $L$ is a constant, we answer this question affirmatively; otherwise, the best we are able to show is that MC-SCHEDULABILITY $\in$ PSPACE. The proof is based on a polynomial-time checkable characterization of an online scheduling policy.

Call a scenario $\left(p_{1}, \ldots, p_{n}\right)$ basic if for each $j=1, \ldots, n$ there exists $\ell_{j} \leq \chi_{j}$ such that $p_{j}=P_{j}\left(\ell_{j}\right)$. Call an on-line scheduling policy basically correct for instance $I$ if for any basic scenario of $I$ the policy generates a feasible schedule. We have the following.

Lemma 1: An instance is MC-schedulable if it admits a basically correct scheduling policy.

Proof: Let $\pi$ be a basically correct policy for instance $I$. We modify $\pi$ to obtain a new policy $\pi^{\prime}$. Policy $\pi^{\prime}$ simulates $\pi$, except that when a job $j$ does not follow a basic scenario (say, $\left.P_{j}(k-1)<p_{j}<P_{j}(k)\right)$, then $\pi^{\prime}$ runs $\pi$ as if job $j$ did not complete before executing $P_{j}(k)$ units. Whenever $\pi$ prescribes the execution of a job that has already signaled completion, $\pi^{\prime}$ idles the processor. The resulting simulated scenario is basic, so $\pi$ feasibly schedules it. Thus $\pi^{\prime}$ feasibly schedules the original scenario (which has the same criticality level). 
In view of Lemma 1, any online policy can be represented as a finite-size decision tree in which each path from the root to a leaf defines the scheduling decisions for a particular scenario. More precisely, a scheduling decision encodes which job should be executed for which amount of time. The decisions are based on when the jobs complete their execution. We show the following lemma, which is crucial for significantly reducing the size of the decision tree for an optimal dynamic policy.

Lemma 2: If an instance is MC-schedulable, then there exists an optimal online scheduling policy that preempts each job $j$ only at time points $t$ such that at time $t$ either some other job is released, or $j$ has executed for exactly $P_{j}(i)$ units of time for some $1 \leq i \leq L$.

Proof: Consider an optimal online scheduling policy that preempts some job $j$ after it has executed for $P_{j}(i)<p<P_{j}(i+1)$ units of time, for some $0 \leq i<L$ (with the convention that $\left.P_{j}(0)=0\right)$. Let $t$ be the first decision time when such a situation occurs, that is, in the optimal decision tree representation this decision is closest to the root for all paths to leaves, and assume $t$ is not the release time of any job. Now, we modify the policy in the following way: we change the decision to preempt $j$ after $p$ units of time into preempting $j$ already after $P_{j}(i)<p$ units of time. Furthermore, at the next decision point (or next release time) $t^{\prime}$, we simply add the remaining amount of processing $p-P_{j}(i)$ to the scheduled amount of processing. Clearly, this modification has to be done for each scenario affected by that change, that is, in the full subtree below the first modified decision. Notice that by preempting $j$ earlier, we do not lose any information on job completions because by Lemma 1 we can assume the scenario is basic. If the original policy completed $j$ no later than its deadline for all scenarios that require this, then this is still true for the modified policy. Moreover, we do not change the amount of execution for jobs that are scheduled between $t$ and $t^{\prime}$. Thus, if the original policy was feasible, then the modified one is feasible as well. Repeated applications of this argument completes the proof.

Theorem 2: The problem of deciding MC-schedulability for $L$ criticality levels is in NP when $L$ is a constant.

Proof: We show that an online policy can be represented and verified in polynomial time and space, if $L$ is constant. Consider an optimal online policy and the corresponding decision tree in which each path from the root to a leaf defines the scheduling decisions for a particular scenario. By Lemma 2, we can assume that the decision times of the policy are those points in time when some job $j$ has completed $P_{j}(i)$ units of time for some $1 \leq i \leq L$. 
Let $N(n, L)$ denote the number of nodes of an optimal decision tree for an instance with $n$ jobs and $L$ criticality levels. We show by induction that $N(n, L)=\mathcal{O}\left(n^{L}\right)$. For $L=1$, there is no decision to be made since the earliest deadline first (EDF) policy is optimal. For larger $L$, any optimal policy starts by executing some job $j$ for some time $P_{j}(i)$. If $j$ completes, we are left with an instance with one less job. If not, the criticality level of the scenario is increased by at least one and thus (by updating the input parameters appropriately) we are left with an instance with $n$ jobs and (at most) $L-1$ levels. Thus, we have the recurrence $N(n, L) \leq N(n-1, L)+N(n, L-1)+1$. Since by induction $N(n, L-1)=\mathcal{O}\left(n^{L-1}\right)$, we have $N(n, L) \leq N(n-1, L)+\mathcal{O}\left(n^{L-1}\right)$, implying $N(n, L)=\mathcal{O}\left(n^{L}\right)$.

Thus, the tree has polynomial size. To verify that the policy is feasible for any possible scenario, we check for each individual path from the root to a leaf if the decisions of the policy lead to a feasible schedule for any scenario compatible with the information extracted by the algorithm. This takes polynomial time.

Theorem 3: The problem of deciding MC-schedulability is in PSPACE.

Proof: Consider the tree representation of an optimal online policy as in the proof of Theorem 2. Notice that we cannot store the whole tree in space that is polynomial in $n$ when $L$ is large. However, we can still check that such a tree exists by generating in depth-first order all paths from the root to a leaf, while making sure that the common portion of consecutive paths is consistent. It is enough to store two paths at a time. Each path requires space proportional to its depth, which is $\mathcal{O}(n L)$, and to keep track of the depth-first search a counter of size $\mathcal{O}(n L)$ is enough, because there are $\mathcal{O}\left(2^{n L}\right)$ potential paths, the tree being binary. Finally, as in the proof of Theorem 2 we verify for each path that the decisions of the policy generate a valid schedule. This yields a nondeterministic algorithm for deciding MC-schedulability that uses polynomial space. The claim follows by the well-known fact that nondeterminism can be removed from the algorithm, at the cost of squaring the required space.

a) Equal deadlines.: Theorem 1 above shows that MC-SCHEDULABILITY is in general NP-hard even if release times are identical. We will now show that the special case in which all jobs have equal deadlines $\left(d_{j}=D, j=1, \ldots, n\right)$ can be solved in polynomial time. We first derive a necessary condition for such an instance $I$ to be MC-schedulable. Consider the criticality level $\ell$ scenario of $I$ in which each job $J_{j}$ needs exactly $p_{j}=P_{j}(\ell)$ execution time. 
Necessary condition: If $I$ is MC-schedulable then for each $\ell$, a scheduling policy exists that allocates to each job $J_{j}$ with $\chi_{j} \geq \ell$ at least $P_{j}(\ell)$ execution time within time window $\left[r_{j}, D\right]$, i.e., the makespan of the scenario is at most $D$.

This condition is easily checked: Let $I_{\ell}=\left\{J_{j} \in I \mid \chi_{j} \geq \ell\right\}$ and $\left|I_{\ell}\right|=n_{\ell}$. Let (after renumbering) $J_{1}, J_{2}, \ldots, J_{n_{\ell}}$ denote the jobs in $I_{\ell}$ in order of non-decreasing release times: $r_{1} \leq$ $r_{2} \leq \ldots \leq r_{n_{\ell}}$. Clearly, the makespan of $I_{\ell}$ is given by

$$
C_{\max }^{\ell}:=\max _{j=1, \ldots, n_{\ell}} r_{j}+\sum_{i=j}^{n_{\ell}} P_{j}(\ell) .
$$

The necessary condition is then verified by checking if

$$
\max _{\ell=1, \ldots, L} C_{\max }^{\ell} \leq D .
$$

Consider the criticality-monotonic (CM) on-line scheduling policy, which schedules at each time instant an available job of highest criticality.

Theorem 4: $\mathrm{CM}$ is correct for all for MC-schedulable instances in which all jobs have the same deadline.

Proof: We prove this by showing that the necessary condition is also sufficient. Consider any scenario of $I$ that has criticality level $\ell$. In a CM-schedule, the scheduling of jobs of criticality $\ell$ or higher is not effected by the presence of lower-criticality jobs, since their execution is postponed as soon as jobs in $I_{\ell}$ become available. Hence, a CM-schedule can be thought of as a schedule that minimizes the makespan of the jobs in $I_{\ell}$. By the necessary condition, this does not exceed the common deadline $D$.

We observe that this theorem also holds when $P_{j}(\ell)$ is not monotonic in $\ell$.

Using essentially the same arguments as were used above for systems with equal deadlines, it can also be shown that

Theorem 5: $\mathrm{CM}$ is correct for all for MC-schedulable instances in which job deadlines are monotonic with criticality level: $\chi_{i}>\chi_{j} \Rightarrow d_{i} \leq d_{j}$.

\section{AlgORITHMS FOR MC SCHEDULING}

Since MC-SCHEDULABILITY is intractable even for dual-criticality instances, we concentrate here on sufficient (rather than exact) MC-schedulability conditions that can be verified in polynomial time. We study two widely-used scheduling policies that yield such sufficient conditions 
and compare their capabilities under the resource augmentation metric: the minimum speed of the processor needed for the algorithm to schedule all instances that are MC-schedulable on a unit-speed processor. We show that the second policy we present outperforms the first one in terms of the resource augmentation metric, in the sense that it needs lower-speed processors to ensure such schedulability.

Run-time support for mixed criticality. In scheduling mixed-criticality systems, the kinds of performance guarantees that can be made depend upon the forms of support that are provided by the run-time environment upon which the system is being implemented. A particularly important form of platform support is the ability to monitor the execution of individual jobs, i.e., being able to determine how long a particular job has been executing.

Why is such a facility useful? In essence, knowledge regarding how long individual jobs have been executing allows the system to become aware, during run-time, when the criticality level of the behavior changes from a value $k$ to the next-higher value $k+1$, due to some job executing beyond its level- $k$ WCET without signalling completion; this information can then be used by the run-time scheduling and dispatching algorithm to no longer execute criticality- $k$ jobs once the transition has occurred.

In the remainder of this section, we assume that this facility to monitor the execution of individual jobs is provided by the run-time environment. We may therefore make the assumption that for each job $J_{j}, P_{j}(\ell)=P_{j}\left(\chi_{j}\right)$ for all $\ell \geq \chi_{j}$. That is, no job executes longer than the WCET at its own specified criticality. This is without loss of generality for any correct scheduling policy: any such policy will immediately interrupt (and no longer schedule) a job $J_{j}$ if its execution time $p_{j}$ exceeds $P_{j}\left(\chi_{j}\right)$, since this makes the scenario of higher criticality level than $\chi_{j}$, and therefore the completion of $J_{j}$ becomes irrelevant for the scenario.

\section{A. Reservations-based scheduling}

As stated in Section I, one straightforward approach is to map each MC job $J_{j}$ into a "traditional" (i.e., non-MC) job with the same arrival time $r_{j}$ and deadline $d_{j}$ and processing time $p_{j}=P_{j}\left(\chi_{j}\right)=\max _{\ell} P_{j}(\ell)$ (by monotonicity), and determine whether the resulting collection of traditional jobs is schedulable using some preemptive single machine scheduling 
algorithm such as the Earliest Deadline First (EDF) rule ${ }^{2}$. This test can clearly be done in polynomial time. We will refer to mixed-criticality instances that are MC-schedulable by this test as worst-case reservations schedulable (WCR-schedulable) instances.

Theorem 6: If an instance is WCR-schedulable on a processor, then it is MC-schedulable on the same processor. Conversely, if an instance $I$ with $L$ criticality levels is MC-schedulable on a given processor, then $I$ is WCR-schedulable on a processor that is $L$ times as fast, and this factor is tight.

Proof: If instance $I$ is WCR-schedulable then for each job the maximum amount of time the job may execute is reserved between its arrival time and its deadline. Hence it is MC-schedulable.

Suppose now that instance $I$ is MC-schedulable. If we were to use a separate processor for each of the $L$ criticality levels, then each job will receive its maximum processing time between arrival time and deadline e.g. by using EDF on the machine corresponding to its criticality level. Hence, by processer sharing, WCR-schedulability on one processor of speed $L$ times faster follows immediately.

Finally, we show that there exist instances with $L$ criticality levels that are MC-schedulable on a given processor, but not WCR-schedulable on a processor that is less than $L$ times as fast.

Consider the instance $I$ comprised of the following $L$ jobs:

$$
\begin{array}{ccc}
J_{1}= & (0,1,1,(1,1, \ldots, 1,1)) \\
J_{2}= & (0,1,2,(0,1, \ldots, 1,1)) \\
\vdots & \vdots \\
J_{L}= & (0,1, L,(0,0, \ldots, 0,1))
\end{array}
$$

This instance is MC-schedulable on a unit-speed processor by the scheduling policy of assigning priority in criticality-monotonic $(\mathrm{CM})$ order: $J_{L}, J_{L-1}, \ldots J_{2}, J_{1}$. Any scenario $\left(p_{1}, p_{2}, \ldots, p_{L}\right)$ with $p_{h}>0, h \geq 2$, and $p_{j}=0$ for all $j>h$, has criticality level $h$, hence all jobs of lower criticality level, in particular $J_{1}$, are not obliged to meet their deadline, and job $h$ will meet its deadline. On the other hand, in any scenario of criticality level $1, p_{2}=p_{3}=\ldots=p_{L}=0$ and $p_{1} \in[0,1]$, hence all jobs meet their deadline.

\footnotetext{
${ }^{2}$ In fact, this approach forms the basis of current practice, as formulated in the ARINC-653 standard: each $J_{j}$ is guaranteed $P_{j}\left(\chi_{j}\right)$ units of execution in a time partitioned schedule, obtained by partitioning the time-line into distinct slots and only permitting particular jobs to execute in each such slot.
} 
However, WCR-schedulability requires that each job $J_{j}$ is executed for $P_{j}\left(\chi_{j}\right)=1, j=$ $1, \ldots, L$ before common deadline 1 , which clearly can only be achieved on a processor with speed at least $L$.

\section{B. Priority-based scheduling}

We now consider another schedulability condition, OCBP-schedulability, that offers a performance guarantee (as measured by the processor speedup factor) that is superior to the performance guarantee offered by the WCR-approach. OCBP-schedulability is a constructive test: we determine off-line, before knowing the actual execution times, a total ordering of the jobs in a priority list and for each scenario execute at each moment in time the available job with the highest priority.

The priority list is constructed recursively using the approach commonly referred to in the real-time scheduling literature as the "Audsley approach" [2], [3]; it is also related to a technique introduced by Lawler [13]. First determine the lowest priority job: Job $J_{i}$ may be assigned the lowest priority if there is at least $P_{i}\left(\chi_{i}\right)$ time between its release time and its deadline available when every other job $J_{j}$ is executed before $J_{i}$ for $P_{j}\left(\chi_{i}\right)$ time units (the WCET of job $J_{j}$ according to the criticality level of job $i$ ). This can be determined by simulating the behavior of the schedule under the assumption that every job other than $J_{i}$ has priority over $J_{i}$ (and ignoring whether these other jobs meet their deadlines or not - i.e., they may execute under any relative priority ordering, and will continue executing even beyond their deadlines). The procedure is repeatedly applied to the set of jobs excluding the lowest priority job, until all jobs are ordered, or at some iteration a lowest priority job does not exist. If job $J_{i}$ has higher priority than job $J_{j}$ we write $J_{i} \triangleright J_{j}$.

Because the priority of a job is based only on its own criticality level, the instance $I$ is called Own Criticality Based Priority OCBP)-schedulable if we find a complete ordering of the jobs.

If at some recursion in the algorithm no lowest priority job exists, we say the instance is not OCBP-schedulable. We can simply argue that this does not mean that the instance is not MCschedulable: Suppose that scheduling according to the fixed priority list $J_{1}, J_{2}, J_{3}$ with $\chi_{2}=1$ and $\chi_{1}=\chi_{3}=2$, proves the instance to be schedulable. It may not be OCBP-schedulable since this does not take into account that $J_{2}$ does not need to be executed at all if $J_{1}$ receives execution time $p_{1}>P_{1}(1)$. 
It is evident that the OCBP priority list for an instance of $n$ jobs can be determined in time polynomial in $n$ : at most $n$ jobs need be tested to determine whether they can be the lowestpriority job; at most $(n-1)$ jobs whether they can be the 2nd-lowest priority jobs; etc. Therefore, at most $x+(n-1)+\cdots+3 \times 2+1=O\left(n^{2}\right)$ simulations need be run, and each simulation takes polynomial time.

We illustrate the operation of the OCBP priority assignment algorithm by an example:

Example 3: Consider the instance comprised of the following three jobs. $J_{1}$ is not subject to certification, whereas $J_{2}$ and $J_{3}$ must be certified correct.

\begin{tabular}{l||lrlll}
$J_{i}$ & $r_{i}$ & $d_{i}$ & $\chi_{i}$ & $P_{i}(1)$ & $P_{i}(2)$ \\
\hline$J_{1}$ & 0 & 4 & 1 & 2 & 2 \\
$J_{2}$ & 0 & 5 & 2 & 2 & 4 \\
$J_{3}$ & 0 & 10 & 2 & 2 & 4
\end{tabular}

Let us determine which, if any, of these jobs could be assigned lowest priority according to the OCBP priority assignment algorithm:

- If $J_{1}$ were assigned lowest priority, $J_{2}$ and $J_{3}$ could consume $P_{2}(1)+P_{3}(1)=2+2=4$ units of processor capacity over $[0,4)$, thus leaving no execution for $J_{1}$ prior to its deadline.

- If $J_{2}$ were assigned lowest priority, $J_{1}$ and $J_{3}$ could consume $P_{1}(2)+P_{3}(2)=2+4=6$ units of processor capacity over $[0,6)$, thus leaving no execution for $J_{2}$ prior to its deadline at time-instant 5 .

- If $J_{3}$ were assigned lowest priority, $J_{1}$ and $J_{2}$ could consume $P_{1}(2)+P_{2}(2)=2+4=6$ units of processor capacity over $[0,6)$. This leaves 4 units of execution for $J_{3}$ prior to its deadline at time-instant 10 , which is sufficient for $J_{3}$ to execute for $P_{3}(2)=4$ time units. Job $J_{3}$ may therefore be assigned the lowest priority.

Next, the OCBP priority assignment algorithm would consider the instance $\left\{J_{1}, J_{2}\right\}$, and seek to assign one of these jobs the lower priority:

- If $J_{1}$ were assigned lower priority, $J_{2}$ could consume $P_{2}(1)=2$ units of processor capacity over $[0,2)$. This leaves 2 units of execution for $J_{1}$ prior to its deadline at time-instant 4 , which is sufficient for $J_{1}$ to execute for $P_{1}(1)=2$ time units. Job $J_{1}$ may therefore be assigned the lowest priority from among $\left\{J_{1}, J_{2}\right\}$.

- It may be verified that $J_{2}$ cannot be assigned the lowest priority from among $\left\{J_{1}, J_{2}\right\}$. If we were to do so, then $J_{1}$ could consume $P_{1}(2)=2$ units of processor capacity over $[0,2)$. 
This leaves 3 units of execution for $J_{1}$ prior to its deadline at time-instant 5 , which is not sufficient for $J_{2}$ to execute for the $P_{2}(2)=4$ time units it needs to complete on time.

The final OCBP priority ordering is therefore as follows. Job $J_{2}$ has the greatest priority, job $J_{1}$ has the next-highest priority, and $J_{3}$ has the lowest priority. It may be verified that scheduling according to these priorities is a correct MC scheduling strategy for the instance $\left\{J_{1}, J_{2}, J_{3}\right\}$, (recall from Section II the definition of "correct" scheduling strategies).

The following theorem shows that the OCBP-test is more powerful than the WCR-test according to the speedup criterion.

Theorem 7: If an instance is OCBP-schedulable on a processor, then it is MC-schedulable on the same processor. Conversely, if instance $I$ with $L$ criticality levels is MC-schedulable on a given processor, then $I$ is OCBP-schedulable on a processor that is $s_{L}$ times as fast, with $s_{L}$ equal to the root of the equation $x^{L}=(1+x)^{L-1}$, and this factor is tight. Furthermore, it holds that $s_{L}=\Theta(L / \ln L)$.

Proof: We present this proof in several parts:

(i) OCBP-schedulability implies MC-schedulability.

(ii) A speedup of $s_{L}$ is sufficient.

(iii) The factor of $s_{L}$ is tight.

(iv) $s_{L}=\Theta(L / \ln L)$.

i): OCBP-schedulability implies $M C$-schedulability. Suppose that $I$ is OCBP-schedulable and suppose, after renumbering jobs, that $J_{1} \triangleright J_{2} \triangleright \cdots \triangleright J_{n}$. Notice that in every behaviour of criticality level $\chi_{k}$, the criticality level of job $J_{k}$, each job $J_{j}$ has $p_{j} \leq P_{j}\left(\chi_{k}\right)$. OCBP-schedulability of $I$ implies that $J_{k}$ can receive $P_{k}\left(\chi_{k}\right)$ units of execution before its deadline if each $J_{i} \in$ $\left\{J_{1}, \ldots, J_{k-1}\right\}$ executes for no more than $P_{i}\left(\chi_{k}\right)$ units.

ii): A speedup of $s_{L}$ is sufficient. Notice that $s_{1}=1$, and that (as one can verify using elementary calculus) $s_{L^{\prime}} \geq s_{L}$ if $L^{\prime}>L$. Let $I$ be an instance with at most $L$ criticality levels that is MC-schedulable on a speed-1 processor, but not OCBP-schedulable on a speed-s processor for some $s \geq s_{L}$, and amongst such instances let it be minimal with respect to $L$ and the number of jobs. Suppose $I$ has $n$ jobs. Minimality of $I$ implies that there is no time-instant $t$ such that $t \notin \cup_{j=1}^{n}\left[r_{j}, d_{j}\right]$, otherwise either the jobs with deadline before $t$ or the jobs with release time after $t$ would comprise a smaller instance with the same property. 
Claim 1: Any job in $I$ with the latest deadline must be of criticality $L$.

Proof: Suppose that a job $J_{i}$ with $\chi_{i}=h<L$ has latest deadline. Create from $I$ an instance $I_{h}$ with level $h$ by "truncating" all jobs with criticality level greater than $h$ to their worst-case level- $h$ scenarios:

$$
\begin{aligned}
J_{j}=\left(r_{j}, d_{j}, \chi_{j},\left(P_{j}(1), \ldots, P_{j}(L)\right)\right) & \in I \rightarrow \\
J_{j}^{\prime} & =\left(r_{j}, d_{j}, \min \left(\chi_{j}, h\right),\left(P_{j}(1), \ldots, P_{j}(h)\right)\right) \in I_{h} .
\end{aligned}
$$

Clearly, $I_{h}$ being a restricted instance of $I$, is MC-schedulable as well, and, by minimality of $I, I_{h}$ is OCBP-schedulable on a speed- $s_{h}$ processor.

That $J_{i}$ has latest deadline in $I$ but cannot be assigned lowest priority on a speed- $s$ processor implies that the scenario with $p_{j}=P_{j}(h)$ cannot be feasibly scheduled on a speed-s processor; thus $I_{h}$ is not clairvoyantly schedulable on a speed-s processor. But $I_{h}$ not being clairvoyantly schedulable implies $I_{h}$ not being OCBP-schedulable, and because $s \geq s_{L} \geq s_{h}$, this contradicts the OCBP-schedulability of $I_{h}$ on a speed- $s_{h}$ processor and completes the proof of the claim.

For each $\ell \in\{1, \ldots, L\}$, let $d(\ell)$ denote the latest deadline of any criticality- $\ell$ job in $I$ : $d(\ell)=\max _{J_{j} \mid \chi_{j}=\ell} d_{j}$. A work-conserving schedule on a processor is a schedule that never leaves the processor idle if there is a job available. Consider any such a work-conserving schedule on a unit-speed processor of all jobs in $I$ of the scenario in which $p_{j}=P_{j}(\ell)$ for all $j$. We define $\Lambda_{\ell}$ as the set of time intervals on which the processor is idle before $d(\ell)$, and $\lambda_{\ell}$ as the total length of this set of intervals.

Claim 2: For each $\ell$ and each $J_{j} \in I$ with $\chi_{j} \leq \ell$ we have $\left[r_{j}, d_{j}\right] \cap \Lambda_{\ell}=\emptyset$.

Proof: Observe that since $s \geq s_{L} \geq 1$, all idle intervals of $\Lambda_{\ell}$ are also idle intervals in any work-conserving schedule of $I$ on a speed-s processor. Hence, any job $J_{j}$ with $\chi_{j} \leq \ell$ with $\left[r_{j}, d_{j}\right] \cap \Lambda_{\ell} \neq \emptyset$ would meet its deadline in such a schedule if it were assigned lowest priority. Since $I$ is assumed to be non-OCBP schedulable on a speed-s processor, this implies that $\left(I \backslash\left\{J_{i}\right\}\right)$ is non-OCBP schedulable on a speed-s processor, contradicting the minimality of $I$. This completes the proof of the claim. It follows that $\Lambda_{L}=\emptyset$ and $\lambda_{L}=0$. 
For each $h=1, \ldots, L$ and $\ell=1, \ldots, L$, let

$$
c_{h}(\ell)=\sum_{J_{j} \mid \chi_{j}=h} P_{j}(\ell)
$$

Notice that by assumption

$$
\forall \ell \forall h \leq \ell: c_{h}(\ell)=c_{h}(h)
$$

Since instance $I$ is clairvoyantly schedulable on a unit-speed processor, clearly we must have

$$
\forall \ell: c_{\ell}(\ell) \leq d(\ell)-\lambda_{\ell}
$$

But also, due to clairvoyant schedulability, the criticality- $\ell$ scenario, in which each job $J_{j}$ with criticality $\geq \ell$ receives exactly $P_{j}(\ell)$ units of execution, completes by the latest deadline $d(L)$ :

$$
\forall \ell: \sum_{i=\ell}^{L} c_{i}(\ell) \leq d(L)-\lambda_{\ell} .
$$

Instance $I$ is not OCBP-schedulable on a speed-s processor, which translated in terms of the introduced notation is:

$$
\forall \ell: \sum_{i=1}^{L} c_{i}(\ell)>s\left(d(\ell)-\lambda_{\ell}\right) .
$$

(This follows from Claim 2, which shows that no job can execute during the idle intervals $\Lambda_{\ell}$. Consequently, all the execution on the jobs must have occurred during the remaining $\left.d(\ell)-\lambda_{\ell}\right)$ time units.)

Hence, for each $\ell$,

$$
\begin{aligned}
s\left(d(\ell)-\lambda_{\ell}\right) & <\sum_{i=1}^{\ell-1} c_{i}(\ell)+\sum_{i=\ell}^{L} c_{i}(\ell) \\
& =\sum_{i=1}^{\ell-1} c_{i}(i)+\sum_{i=\ell}^{L} c_{i}(\ell) \quad(\text { by }(3)) \\
& \leq \sum_{i=1}^{\ell-1}\left(d(i)-\lambda_{i}\right)+\left(d(L)-\lambda_{\ell}\right) \quad(\text { by (4) and }(5)) \\
& \leq \sum_{i=1}^{\ell-1}\left(d(i)-\lambda_{i}\right)+d(L) .
\end{aligned}
$$

Therefore, for all $\ell=1, \ldots, L$,

$$
s<\frac{d(L)+\sum_{i=1}^{\ell-1}\left(d(i)-\lambda_{i}\right)}{d(\ell)-\lambda_{\ell}}
$$


Using notation $\delta_{\ell}=d(\ell)-\lambda_{\ell}$ (hence $\delta_{L}=d(L)$ since $\lambda_{L}=0$ ) this yields

$$
s<\min _{\ell=1, \ldots, L} \frac{\delta_{L}+\sum_{i=1}^{\ell-1} \delta_{i}}{\delta_{\ell}}
$$

The minimum is maximized if all $L$ terms are equal. Let $x$ be this maximum value. Then for all $\ell=1, \ldots, L$,

$$
x=\frac{\delta_{L}+\delta_{1}+\delta_{2}+\cdots+\delta_{\ell-1}}{\delta_{\ell}}=\frac{x \delta_{\ell-1}+\delta_{\ell-1}}{\delta_{\ell}}=\left(\frac{1+x}{\delta_{\ell}}\right) \delta_{\ell-1} .
$$

Hence,

$$
\delta_{\ell}=\left(\frac{1+x}{x}\right) \delta_{\ell-1} \forall \ell=1, \ldots, L \quad \text { which implies } \quad \delta_{L}=\left(\frac{1+x}{x}\right)^{L-1} \delta_{1} .
$$

Since, in particular, $x=\frac{\delta_{L}}{\delta_{1}}$, we have

$$
x=\left(\frac{1+x}{x}\right)^{L-1}
$$

which concludes the proof that a speedup of $s_{L}$ is sufficient.

iii): The factor of $s_{L}$ is tight. We now show that the factor $s_{L}$ is tight by giving instances with $L$ criticality levels that are MC-schedulable on a unit-speed processor, but not OCBP-schedulable on a processor that is less than $s_{L}$ times as fast.

Consider the following instance consisting of $2 L-1$ jobs:

- $J_{1}=\left(0, d_{1}=\sigma_{1}=1,1,(\overbrace{1,1, \ldots, 1}^{L \text { times }})\right)$.

- For each $i, 2 \leq i \leq L$, there are two jobs:

$$
\begin{aligned}
& \text { - } J_{2(i-1)}=\left(0, \sigma_{i-1}, i,(\overbrace{0,0, \ldots, 0}^{(i-1) \text { times }}, \underbrace{\sigma_{i-1}, \ldots, \sigma_{i-1}}_{L-(i-1) \text { times }})\right) \\
& \text { - } J_{2 i-1}=\left(0, \sigma_{i}, i,(\underbrace{\sigma_{i}-\sigma_{i-1}, \ldots, \sigma_{i}-\sigma_{i-1}}_{L \text { times }})\right) \text {, where } \sigma_{i}>\sigma_{i-1} \text {. }
\end{aligned}
$$

This instance is MC-schedulable by the following policy. Assign greatest priority to the jobs $J_{2 i}$ in reverse order of their indices: $J_{2 L}, J_{2(L-1)}, \ldots, J_{2}$. Consider the scenario in which $p_{2 h}>0$, $h \geq 1$, and $p_{2 j}=0, j>h$. Then we execute $J_{2 h}, J_{2 h+1}, J_{2 h+3}, \ldots, J_{2 L+1}$ in this order; it is evident that each of them completes by its deadline.

For job $J_{2 h-1}, h=1, \ldots, L$ to be assigned lowest priority in an OCBP-schedule, we would need a speedup factor $s$ of the processor such that

$$
\begin{aligned}
\frac{\left(\sigma_{L}-\sigma_{L-1}\right)+\left(\sigma_{L-1}-\sigma_{L-2}\right)+\cdots+\left(\sigma_{2}-\sigma_{1}\right)+\sigma_{1}+\left(1+\sigma_{2}+\cdots+\sigma_{j-1}\right)}{s} & = \\
\frac{\sigma_{L}+\left(1+\sigma_{2}+\cdots+\sigma_{j-1}\right)}{s} & \leq \sigma_{h} .
\end{aligned}
$$


Hence, for all $h=1, \ldots, L$, it requires

$$
s \geq \frac{\sigma_{L}+\left(1+\sigma_{2}+\cdots+\sigma_{j-1}\right)}{\sigma_{h}} .
$$

We refer to the end of part (ii) of the proof to show that the right hand side is maximized for the root of the equation $x^{L}=(1+x)^{L-1}$.

iv): $\mathbf{s}_{\mathbf{L}}=\boldsymbol{\Theta}(\mathbf{L} / \ln \mathbf{L})$. Rewrite the equation as $x=(1+1 / x)^{L-1}$ and let $x^{*}$ be its largest real root. The left hand side (resp., r.h.s.) is increasing (resp., decreasing) in $x$. The 1.h.s. is larger (resp., smaller) than the r.h.s. precisely when $x>x^{*}$ (resp., $x<x^{*}$ ). So if substituting (say) $f(L)$ in place of $x$ gives a 1.h.s. larger (resp., smaller) than the r.h.s., it means that $f(L)$ is an upper (resp., lower) bound on $x^{*}$.

Substituting $2(L-1) / \ln L$ in place of $x$, we get for the r.h.s.:

$$
(1+1 / x)^{L-1} \leq e^{(L-1) / x}=e^{(L-1)(\ln L) / 2(L-1)}=L^{1 / 2}
$$

(where we have used $1+y \leq e^{y}$ ). The 1.h.s. becomes instead $2(L-1) / \ln L$, which is larger than the r.h.s. for all $L \geq 2$. So $x^{*} \leq 2(L-1) / \ln L$ for all $L \geq 2$.

Substituting $(L-1) /(2 \ln L)$ in place of $x$, we get for the r.h.s.:

$$
(1+1 / x)^{L-1} \geq e^{(L-1) \frac{1}{2 x}}=L
$$

(where we have used $1+2 y \geq e^{y}$ for all $y \in[0,1.2]$, and assumed $L \geq 3$ ). The 1.h.s. becomes instead $(L-1) /(2 \ln L)$, which is smaller than the r.h.s. for all $L \geq 2$. So $x^{*} \geq(L-1) /(2 \ln L)$ for all $L \geq 3$.

We note that for $L=2$ in the above theorem, $s_{2}=(1+\sqrt{5}) / 2$ is equal to the golden ratio $\phi$; thus the result is a true generalization of an earlier result in [6]. In general, $s_{L}=$ $\Theta(L / \ln L)$; hence, this priority-based scheduling approach asymptotically improves on the worstcase reservations-based approach by a factor of $\Theta(\ln L)$ from the perspective of processor speedup factors.

Notice that the proof of the speedup bound for OCBP-schedulability in Theorem 7 only uses the clairvoyant-schedulability of the instance, which is a weaker condition than MCschedulability (recall Proposition 1). Moreover, Proposition 1 shows that it is not possible to get an improved test if the proof of its speedup bound is based on clairvoyant-schedulability alone. 
Nevertheless, the question remains if a test other than OCBP can test MC-schedulability within a smaller speedup bound. We do not give a full answer to this question. However, we can rule out fixed-priority policies, that is, policies which execute the jobs in some ordering fixed before execution. This ordering is not adapted during execution, except that we do not execute jobs of criticality level $i<h$ after a scenario was revealed to be a level- $h$ scenario. Such a policy admits a simple representation as a sequence of jobs and we say that an instance $I$ is $\Pi$-schedulable if there exists an ordering of jobs $\Pi$ that is feasible for for any non-erroneous scenario.

The following result shows that OCBP is best possible among fixed-priority policies.

Theorem 8: There exist instances with $L$ criticality levels that are clairvoyantly-schedulable, but that are not $\Pi$-schedulable for any fixed priority policy $\Pi$ on a processor that is less that $s_{L}$ times as fast, with $s_{L}$ being the root of the equation $x^{L}=(1+x)^{L-1}$.

Proof: Consider an instance with $L$ criticality levels and $L$ jobs:

$$
J_{1}:(0,1,1,(\overbrace{1,1, \ldots, 1}^{L \text { times }})) \text {, }
$$

and, for each $i=2, \ldots, L$,

$$
J_{i}:\left(0, \sigma_{i}, i,(\overbrace{\sigma_{i}-\sigma_{i-1}, \ldots, \sigma_{i}-\sigma_{i-1}}^{i-1 \text { times }}, \overbrace{\sigma_{i}, \ldots, \sigma_{i}}^{L-i+1})\right)
$$

where $\sigma_{i}$ will be specified later and satisfies $\sigma_{i-1}<\sigma_{i}$.

For $L=3$ we have the following example:

$$
\begin{aligned}
& J_{1}:(0, \quad 1, \quad 1, \quad(1, \quad 1, \quad 1)) \\
& J_{2}:\left(0, \quad \sigma_{2}, 2, \quad\left(\sigma_{2}-1, \quad \sigma_{2}, \quad \sigma_{2}\right)\right) \\
& J_{3}:\left(0, \quad \sigma_{3}, \quad 3, \quad\left(\sigma_{3}-\sigma_{2}, \quad \sigma_{3}-\sigma_{2}, \sigma_{3}\right)\right) \text {. }
\end{aligned}
$$

The system is clairvoyantly schedulable as, for each scenario of level $i$ and for each job $J_{j}$, $j \geq i, \sum_{\ell=i}^{j} P_{\ell}(i)=\sigma_{j}$. It follows that a schedule that executes job $J_{i}$ in the interval $\left[0, \sigma_{i}\right]$ and each job $J_{j}, j>i$, in the interval $\left[\sigma_{j-1}, \sigma_{j}\right]$ is feasible.

We now show that the system is $\Pi$-schedulable for a $s$-speed machine only if $s \geq s_{L}$ where $s_{L}$ is the positive real-valued solution of the equation

$$
x^{L}=(x+1)^{L-1} .
$$

Each fixed priority work-conserving policy is a sequence of jobs. Let us consider a sequence where the last scheduled job is $J_{i}$ and a level $i$ scenario. In this case the overall execution time 
is $\sum_{j=1}^{L} P_{j}(i)=\sigma_{L}+\sum_{j=1}^{i-1} \sigma_{j}$. Hence the schedule is feasible for a $s$-speed machine if and only if:

$$
s \sigma_{i} \geq \sigma_{L}+\sum_{j=1}^{i-1} \sigma_{j} .
$$

By using the same arguments for each possible schedule, it follows that a fixed priority policy $\Pi$ system is correct for a $s$-speed machine if and only if

$$
s \geq \min _{1 \leq i \leq L}\left\{\frac{\sigma_{L}+\sum_{j=1}^{i-1} \sigma_{j}}{\sigma_{i}}\right\} .
$$

As we showed in the proof of Theorem $7, s_{L}$ is the maximum value of $s^{\prime}$ satisfying the inequality:

$$
\min _{1 \leq i \leq L}\left\{\frac{\sigma_{L}+\sum_{j=1}^{i-1} \sigma_{j}}{\sigma_{i}}\right\} \geq s^{\prime},
$$

hence the system is $\Pi$-schedulable for a $s$-speed machine if and only if $s \geq s_{L}$.

\section{MC-SChEDUlability With BOUNDED WCET}

Although Theorems 7 and 8 above provide tight bounds on the performance of OCBP and similar algorithms, better performance than is implied by these bounds may be possible when further restrictions are placed on the kinds of instances that could need to be scheduled. We illustrate this phenomenon in this section, by analyzing the OCBP scheduling policy upon dualcriticality instances when the overall WCET at level two of criticality-two jobs is a priori known to be bounded by a constant times the level-one WCET's of these jobs. That is, let $c_{1}=$ $\sum_{j \mid \chi_{j}=1} P_{j}(1)$ denote the cumulative WCET for jobs with criticality level 1 , and let $c_{2}(1)=$ $\sum_{j \mid \chi_{j}=2} P_{j}(1)$ and $c_{2}(2)=\sum_{j \mid \chi_{j}=2} P_{j}(2)$ denote the cumulative WCETs for jobs with criticality level 2 at levels 1 and 2 , respectively. We consider the situation that $c_{2}(2) \leq \beta c_{2}(1)$ for a certain constant $\beta$, whose value is known $^{3}$.

The following theorem shows that if $\beta<1+\phi, \phi=(1+\sqrt{5}) / 2$, the speed required by OCBP to give a necessary condition for MC-schedulability is smaller than the bound of Theorem 7.

\footnotetext{
${ }^{3}$ This would hold in, for instance, systems in which the higher-criticality-level WCET of each job is no more than $\beta$ times the lower-criticality-level WCET of that job. That is, the constant $\beta$ could simply be the maximum degree of pessimism exhibited by the WCET-analysis tool used in the higher criticality level as compared to the tool used in the lower criticality level.
} 
Theorem 9: If an instance $I$ with 2 criticality levels is MC-schedulable on a given processor and $c_{2}(2) \leq \beta c_{2}(1)$ for a certain constant $\beta$, then $I$ is OCBP-schedulable on a processor that is $s$ times as fast, where $s$ is given by

$$
s= \begin{cases}\frac{\sqrt{\beta^{2}+\beta+1}+\beta}{\beta+1} & \text { if } \beta \leq 1+\phi \\ \phi & \text { if } \beta>1+\phi .\end{cases}
$$

Proof: Let $I$ be a minimal instance that is MC-schedulable on a given processor and not OCBP-schedulable on a processor that is $s$ times as fast for some $s>1$. We show that

$$
s< \begin{cases}\frac{\sqrt{\beta^{2}+\beta+1}+\beta}{\beta+1} & \text { if } \beta \leq 1+\phi \\ \phi & \text { if } \beta>1+\phi .\end{cases}
$$

Let $d_{1}$ and $d_{2}$ denote the latest deadlines at level 1 and 2, respectively, and let $j_{1}$ and $j_{2}$ be the jobs with deadlines $d_{1}$ and $d_{2}$. Recall from the first claim in the proof of Theorem 7 that the jobs in $I$ with latest deadline must be of criticality 2. Define $\lambda_{1}$ as in the proof of Theorem 7. That is, consider any work-conserving schedule on a unit-speed processor of all jobs in $I$ of the scenario in which $p_{j}=P_{j}(1)$ for all $j: \lambda_{1}$ is defined to be the total amount of time during which the processor is idle before $d_{1}$.

Since $I$ is MC-schedulable then $c_{1}$ cannot exceed $d_{1}$ in any criticality 1 scenario. Moreover, in scenarios where all jobs execute for their WCET at criticality $1, c_{1}+c_{2}(1)$ cannot exceed $d_{2}$ and in scenarios where all jobs execute for their WCET at criticality 2, $c_{2}(2)$ cannot exceed $d_{2}$. Hence, by instantiating Inequality 4 for $\ell=1$ we have

$$
c_{1} \leq\left(d_{1}-\lambda_{1}\right)
$$

By instantiating Inequality 5 for $\ell=1$ and noting that $\left(d_{2}-\lambda_{1}\right) \leq d_{2}$ we have

$$
c_{1}+c_{2}(1) \leq d_{2}
$$

By instantiating Inequality 4 for $\ell=2$ and noting that $\lambda_{2}=0$ for dual-criticality systems $(L=2)$ we have

$$
c_{2}(2) \leq d_{2} .
$$

Since $I$ is not OCBP-schedulable on a speed- $s$ processor, $j_{1}$ and $j_{2}$ cannot be the lowest priority jobs on such a processor. Hence by instantiating Inequality 6 for $\ell=1$ we have

$$
c_{1}+c_{2}(1)>s\left(d_{1}-\lambda_{1}\right)
$$


Similarly, by instantiating inequality (6) for $\ell=2$ and noting that $\lambda_{2}=0$ for dual-criticality systems $(L=2)$ we have

$$
c_{1}+c_{2}(2)>s d_{2}
$$

As $c_{2}(2) \leq \beta c_{2}(1)$, inequality (12) implies

$$
c_{1}+\beta c_{2}(1)>s d_{2}
$$

We analyze the following two cases.

Case $i): \beta c_{2}(1) \leq d_{2}$.

In this case we have

$$
\frac{\beta^{2} c_{2}(1)}{\beta+1} \leq \frac{\beta d_{2}}{\beta+1} .
$$

Multiplying (8) by $\frac{1}{\beta+1}$, (9) by $\frac{\beta}{\beta+1}$ and adding them to (14) yields:

$$
c_{1}+\beta c_{2}(1) \leq \frac{1}{\beta+1}\left(d_{1}-\lambda_{1}\right)+\frac{2 \beta}{\beta+1} d_{2}
$$

This together with (13) implies

$$
s d_{2}<\frac{1}{\beta+1}\left(d_{1}-\lambda_{1}\right)+\frac{2 \beta}{\beta+1} d_{2} .
$$

which rewritten using $x=\frac{\left(d_{1}-\lambda_{1}\right)}{d_{2}}$ is

$$
s<\frac{1}{\beta+1} x+\frac{2 \beta}{\beta+1} .
$$

By (9) and (11) we have $s\left(d_{1}-\lambda_{1}\right)<d_{2}$ and hence $x s d_{2}<d_{2}$, implying that $s<\frac{1}{x}$. Hence

$$
s<\min \left\{\frac{1}{\beta+1} x+\frac{2 \beta}{\beta+1}, \frac{1}{x}\right\} .
$$

The largest value of $s$ satisfying the above inequality is

$$
s=\frac{\sqrt{\beta^{2}+\beta+1}+\beta}{\beta+1} .
$$

Case $i i): \beta c_{2}(1)>d_{2}$.

Inequality (9) and $c_{2}(1)>\frac{d_{2}}{\beta}$ imply $c_{1}<d_{2}-\frac{d_{2}}{\beta}$ which together with inequality (12) implies

$$
d_{2}-\frac{d_{2}}{\beta}+c_{2}(2)>s d_{2}
$$

As $c_{2}(2) \leq d_{2}$, we have $d_{2}-\frac{d_{2}}{\beta}+d_{2}>s d_{2}$ and hence

$$
s<2-\frac{1}{\beta} \text {. }
$$




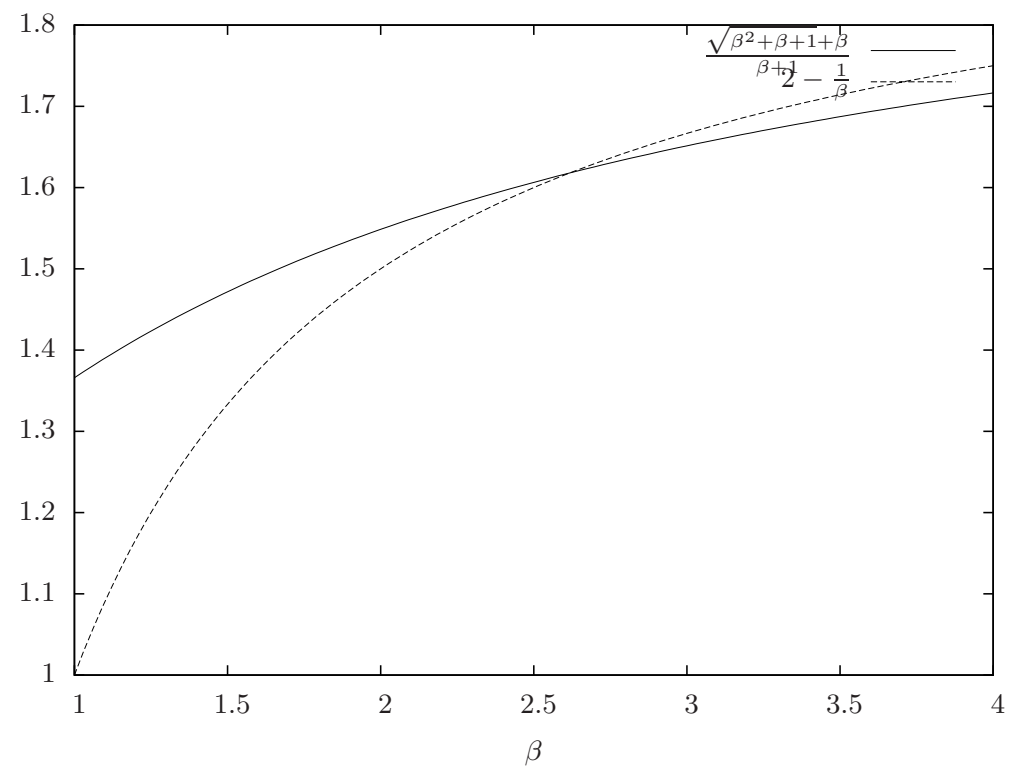

Fig. 1. Upper bounds for cases (i) and (ii).

See Figure 1 for a plot of the upper bounds given by cases $i$ ) and $i$ ) as a function of $\beta$. The upper bounds are monotonically non-increasing with $\beta$ and both of them are equal to $\phi$ for $\beta=1+\phi$. Moreover, the upper bound given by case $i$ ) is greater that the upper bound given by case (ii) for each $\beta<1+\phi$. Hence, by taking the worst of the above cases, for $\beta<1+\phi$ we obtain a better bound for OCBP. In detail:

$$
s< \begin{cases}\frac{\sqrt{\beta^{2}+\beta+1}+\beta}{\beta+1} & \text { if } \beta \leq 1+\phi \\ \phi & \text { if } \beta>1+\phi\end{cases}
$$

\section{RELATED WORK}

As we have stated in Section I, the strategic significance of mixed criticality certification is widely recognized and has been the subject of multiple workshops and working-group meetings, some of the findings of which are highlighted in a white paper [4]. To our knowledge, the scheduling problem that arises from multiple certification requirements, at different criticality levels, was first identified and formalized by Vestal in [15], in the context of the fixed-priority preemptive uniprocessor scheduling of recurrent task systems. 
Although many other real-time scheduling papers deal with mixed-criticality systems, they do not really deal with scheduling for certification. Lakshmanan et al. [8], [11], [12] deal with a different aspect of mixed-criticality systems from the one we focus on here, in that they do not directly address the certification issue. Nevertheless, [8], [11], [12] contain very interesting and novel ideas that merit mention. This body of work observes that the complete inter-criticality isolation offered by the reservations approach may cause criticality inversion: preventing a higher-criticality job from meeting its deadline while allowing lower-criticality jobs to complete. On the other hand, assigning priorities according to criticality may result in very poor processor utilization. An innovative slack-aware approach is proposed that builds atop priority-based scheduling (with priorities not necessarily assigned according to criticality), to allow for asymmetric protection of reservations thereby helping to lessen criticality inversion while retaining reasonable resource utilization. The schedulability guarantee that their proposed algorithm can make may be stated as follows in the context of systems with only two criticality levels: all deadlines of high-criticality tasks are guaranteed to be met regardless of the run-time behavior of the lower-criticality tasks, provided the execution of at most one higher-criticality job overruns its WCET estimated at the lower criticality level (to no more than its WCET estimated at a level of assurance consistent with the higher criticality level). Although this represents a significant improvement over prior approaches, it is far removed from what would pass certification: for that, we would need to guarantee that all higher-criticality tasks complete by their deadlines even if they all execute for up to their WCET requirements specified at the higher level of assurance.

Pellizzoni et al. [14], use a reservations-based approach to ensure strong isolation among sub-systems of different criticalities; this paper proposes innovative design and architectural techniques for preserving such isolation despite some necessary interaction (e.g., in the sharing of additional non-preemptable resources) between jobs of different criticalities. The focus differs from ours in that the goal is not to optimize resource utilization, but to ensure isolation amongst the different criticalities.

\section{CONCLUSIONS}

As safety-critical real-time embedded systems become larger and more complex, the cost and complexity of obtaining certification for such systems is becoming a serious concern [4]. In 
mixed-criticality systems, these certification considerations give rise to fundamental new resource allocation and scheduling challenges; results from conventional real-time scheduling theory do not appear to be adequate for dealing with these challenges.

The research described in this document represents our efforts at devising new formal models, analysis, and algorithms for mixed-criticality scheduling. We have described a model for representing a simple kind of mixed-criticality system - those that can be represented as collections of independent jobs executing upon a single preemptable processor. We have studied the complexity of MC-SCHEDUlability: determining whether a given mixed-criticality instance, specified according to our model, is schedulable or not. We show that this is an NP-hard problem. For instances with a fixed (constant) number of criticality levels, it is NP-complete. We have also shown that the problem is in PSPACE for instances with arbitrarily many distinct criticality levels, but leave establishing the precise complexity as an open problem.

We gave an example of a special case of MC-SCHEDULABILITY that is solvable in polynomial time: when all the jobs have the same deadline. It is left for future research to investigate the boundaries between hardness and well-solvability further.

We have also provided a framework for comparing the powerfulness of MC-schedulability analysis algorithms via the metric of the speed-up factor. We have proposed a mixed-criticality scheduling algorithm (OCBP), and have quantified its performance guarantees according to the speedup-factor. We conjecture that the OCBP-schedulability test is the best possible in terms of the speedup factor metric, but we have only been able to prove this within a restricted class of scheduling policies.

\section{REFERENCES}

[1] ARINC. ARINC 653-1 Avionics application software standard interface, October 2003.

[2] N. C. Audsley. Optimal priority assignment and feasibility of static priority tasks with arbitrary start times. Technical report, The University of York, England, 1991.

[3] N. C. Audsley. Flexible Scheduling in Hard-Real-Time Systems. PhD thesis, Department of Computer Science, University of York, 1993.

[4] J. Barhorst, T. Belote, P. Binns, J. Hoffman, J. Paunicka, P. Sarathy, J. S. P. Stanfill, D. Stuart, and R. Urzi. White paper: A research agenda for mixed-criticality systems, April 2009. Available at http://www.cse.wustl.edu/ cdgill/CPSWEEK09_MCAR.

[5] S. Baruah, H. Li, and L. Stougie. Mixed-criticality scheduling: improved resource-augmentation results. In Proceedings of the ICSA International Conference on Computers and their Applications (CATA). IEEE, April 2010. 
[6] S. Baruah, H. Li, and L. Stougie. Towards the design of certifiable mixed-criticality systems. In Proceedings of the IEEE Real-Time Technology and Applications Symposium (RTAS). IEEE, April 2010.

[7] S. K. Baruah, V. Bonifaci, G. D’Angelo, H. Li, A. Marchetti-Spaccamela, N. Megow, and L. Stougie. Scheduling realtime mixed-criticality jobs. In P. Hlinený and A. Kucera, editors, Proceedings of the 35th International Symposium on the Mathematical Foundations of Computer Science, volume 6281 of Lecture Notes in Computer Science, pages 90-101. Springer, 2010.

[8] D. de Niz, K. Lakshmanan, and R. R. Rajkumar. On the scheduling of mixed-criticality real-time task sets. In Proceedings of the Real-Time Systems Symposium, pages 291-300, Washington, DC, 2009. IEEE Computer Society Press.

[9] M. Garey and D. Johnson. Computers and Intractability : a Guide to the Theory of NP-Completeness. W. H. Freeman and company, NY, 1979.

[10] B. Kalyanasundaram and K. Pruhs. Speed is as powerful as clairvoyance. Journal of the ACM, 37(4):617-643, 2000.

[11] K. Lakshmanan, D. de Niz, and R. R. Rajkumar. Mixed-criticality task synchronization in zero-slack scheduling. In Proceedings of the Real-Time and Embedded Technology and Applications Symposium, Chicago, 2011. IEEE Computer Society Press.

[12] K. Lakshmanan, D. de Niz, R. R. Rajkumar, and G. Moreno. Resource allocation in distributed mixed-criticality cyberphysical systems. In Proceedings of the 30th International Conference of Distributed Computing Systems. IEEE Computer Society Press, 2010.

[13] E. L. Lawler. Optimal sequencing of a single machine subject to precedence constraints. Management Science, 19(5):544546, 1973.

[14] R. Pellizzoni, P. Meredith, M. Y. Nam, M. Sun, M. Caccamo, and L. Sha. Handling mixed criticality in SoC-based real-time embedded systems. In Proceedings of the International Conference on Embedded Software (EMSOFT), Grenoble, France, 2009. IEEE Computer Society Press.

[15] S. Vestal. Preemptive scheduling of multi-criticality systems with varying degrees of execution time assurance. In Proceedings of the Real-Time Systems Symposium, pages 239-243, Tucson, AZ, December 2007. IEEE Computer Society Press.

[16] J. Windsor and K. Hjortnaes. Time and space partitioning in spacecraft avionics. Space Mission Challenges for Information Technology, IEEE International Conference on, pages 13-20, 2009. 